\title{
Early science with the Large Millimetre Telescope: Deep LMT/AzTEC millimetre observations of $\epsilon$ Eridani and its surroundings
}

\author{
M. Chavez-Dagostino, ${ }^{1 \star}$ E. Bertone,${ }^{1 \star}$ F. Cruz-Saenz de Miera, ${ }^{1}$ J. P. Marshall,,${ }^{2,3 \star}$ \\ G. W. Wilson, ${ }^{4}$ D. Sánchez-Argüelles, ${ }^{1}$ D. H. Hughes, ${ }^{1}$ G. Kennedy, ${ }^{5}$ O. Vega, ${ }^{1}$ \\ V. De la Luz, ${ }^{6}$ W. R. F. Dent, ${ }^{7}$ C. Eiroa ${ }^{8,9}$ A. I. Gómez-Ruiz, ${ }^{1}$ J. S. Greaves ${ }^{10}$ \\ S. Lizano, ${ }^{11}$ R. López-Valdivia, ${ }^{1}$ E. Mamajek, ${ }^{12}$ A. Montaña, ${ }^{1}$ M. Olmedo, ${ }^{1}$ \\ I. Rodríguez-Montoya, ${ }^{1}$ F. P. Schloerb, ${ }^{4}$ Min S. Yun, ${ }^{4}$ J. A. Zavala ${ }^{1}$ and M. Zeballos ${ }^{1}$ \\ ${ }^{1}$ Instituto Nacional de Astrofísica Optica y Electrónica Luis Enrique Erro \#1, CP 72840, Tonantzintla, Puebla, México \\ ${ }^{2}$ School of Physics, University of New South Wales, Sydney, NSW 2052, Australia \\ ${ }^{3}$ Australian Centre for Astrobiology, University of New South Wales, Sydney, NSW 2052, Australia \\ ${ }^{4}$ Department of Astronomy, University of Massachusetts, Amherst, MA 01003, USA \\ ${ }^{5}$ Institute of Astronomy, University of Cambridge, Cambridge CB3 OHA, UK \\ ${ }^{6}$ SciESMEX, Instituto de Geofísica, Unidad Michoacán, Universidad Nacional Autónoma de México, Antigua Carretera a Pátzcuaro 8701, \\ Morelia, Michoacán, CP 58089, México \\ ${ }^{7}$ ALMA SCO, Alonso de Córdova 3107, Vitacura, Casilla 763 0355, Santiago, Chile \\ ${ }^{8}$ Departamento de Física Teórica, C-XI, Facultad de Ciencias, Universidad Autónoma de Madrid, Canto Blanco E-28049, Madrid, Spain \\ ${ }^{9}$ Astro-UAM, Unidad Asociada UAM - CSIC, Madrid, Spain \\ ${ }^{10}$ School of Physics and Astronomy, Cardiff University, Queen's Buildings, The Parade, Cardiff CF24 3AA, UK \\ ${ }^{11}$ Instituto de Radio Astronomía y Astrofísica, Universidad Nacional Autónoma de México, Antigua Carretera a Pátzcuaro 8701, \\ Morelia, Michoacán, CP 58089, México \\ ${ }^{12}$ Department of Physics and Astronomy, University of Rochester, Rochester, NY 14627-0171, USA
}

Accepted 2016 June 2. Received 2016 June 2; in original form 2016 March 11

\begin{abstract}
$\epsilon$ Eridani is a nearby, young Sun-like star that hosts a ring of cool debris analogous to the Solar system's Edgeworth-Kuiper belt. Early observations at (sub-)mm wavelengths gave tentative evidence of the presence of inhomogeneities in the ring, which have been ascribed to the effect of a putative low eccentricity planet, orbiting close to the ring. The existence of these structures has been recently challenged by high-resolution interferometric millimetre observations. Here, we present the deepest single-dish image of $\epsilon$ Eridani at millimetre wavelengths, obtained with the Large Millimetre Telescope Alfonso Serrano (LMT). The main goal of these LMT observations is to confirm (or refute) the presence of non-axisymmetric structure in the disc. The dusty ring is detected for the first time along its full projected elliptical shape. The radial extent of the ring is not spatially resolved and shows no evidence, to within the uncertainties, of dust density enhancements. Additional features of the $1.1 \mathrm{~mm}$ map are: (i) the presence of significant flux in the gap between the ring and the star, probably providing the first exo-solar evidence of Poynting-Robertson drag, (ii) an unambiguous detection of emission at the stellar position with a flux significantly above that expected from $\epsilon$ Eridani's photosphere, and (iii) the identification of numerous unresolved sources which could correspond to background dusty star-forming galaxies.
\end{abstract}

Key words: circumstellar matter-stars: individual: $\epsilon$ Eridani-submillimetre: stars.

\section{INTRODUCTION}

The circumstellar debris discs detected around mature, mainsequence stars are a visible remnant of planet formation processes (Backman \& Paresce 1993). Composed of icy and rocky bodies ranging from micron-sized grains to kilometre-sized planetesimals,
^E-mail: mchavez@inaoep.mx (MC-D); ebertone@inaoep.mx (EB); jonty.marshall@unsw.edu.au (JPM) 
the presence of a disc is typically revealed through the detection of excess emission from the star at mid- and far-infrared (FIR) wavelengths (Wyatt 2008; Matthews et al. 2014).

Recent surveys by the Herschel Space Observatory (Pilbratt et al. 2010) have identified cool discs, analogues to the EdgeworthKuiper belt of our Solar system, in around $20 \pm 2$ per cent of Sun-like stars (Eiroa et al. 2013). However, their detection rate depended on both the temperature of the host star and the observing strategy of the space craft. For instance, Thureau et al. (2014) found an incidence of 30 per cent in A-type stars. The combination of exoplanet and debris disc surveys has provided evidence that planets are more common around stars that also host a debris disc (Bryden et al. 2013), and revealed tentative correlations linking the presence of dust and planets with the properties of the host star (Wyatt et al. 2012; Maldonado et al. 2012; Marshall et al. 2014a; MoroMartín et al. 2015). The presence of a planet around a host star can be revealed through its dynamical interaction with the debris disc which creates non-axisymmetric structures (clumps, warps, cavities, etc.) in the disc. Such structures led to the discovery of a giant planetary companion in the prototypical debris disc host $\beta$ Pictoris (Lagrange et al. 2010). Thermal emission from the micron-sized dust grains dominates the observed flux of the disc at FIR wavelengths, and exhibits typical temperatures of 30 to $80 \mathrm{~K}$ (Morales et al. 2011) and radial size scales of $10 \mathrm{~s}$ to $100 \mathrm{~s}$ of astronomical units (au) (Pawellek et al. 2014, see also Marshall et al. 2014a). Tracing the largest millimetre-sized and coolest grains in the disc, which do not drift as far from their parent planetesimal belt under the action of radiation forces as the smaller micron-sized grains (Krivov et al. 2008), is vital to accurately determine the location of the dust-producing belt of planetesimals around the star (Krivov 2010). Such measurements are only possible with (sub-)millimetre continuum imaging observations (e.g. Williams \& Andrews 2006; Nilsson et al. 2010; Panić et al. 2013). Resolving the radial extent of the disc is fundamental in the modelling process as it directly constrains the orbital radius of the dust responsible for the observed emission, weakening inherent degeneracies between grain size and radial distance in those models reliant solely on the disc thermal emission derived from the analyses of the spectral energy distribution (SED; Augereau et al. 1999; Lebreton et al. 2012; Ertel et al. 2014; Marshall et al. 2014b).

$\epsilon$ Eridani ('Ran', HR1084, HD22049, HIP16537) is a relatively young (age $=0.8 \mathrm{Gyr}$; Di Folco et al. 2004; Mamajek \& Hillenbrand 2008; 1.4 Gyr, Bonfanti et al. 2015), nearby $(d=3.22 \mathrm{pc})$ Sun-like (spectral class K2V) star. Its age and distance place it as the closest isolated star of this kind where we can study the early stages in the evolution of a planetary system analogous to the Solar system. The star is host to a bright, extended, almost face-on debris disc, which ranks amongst the finest examples of these objects so far discovered (Greaves et al. 1998; Holland et al. 1998). Recent models (Backman et al. 2009; Reidemeister et al. 2011) suggest that the disc comprised up to four distinct components: two warm inner belts, a cold outer belt and an extended halo of small grains. In these models the dust in the warm components actually originates in the cold belt and is transported to the inner regions through the Poynting-Robertson drag and stellar winds. Radial velocity analyses suggest the existence of two giant exoplanets in addition to the warm inner debris disc (Hatzes et al. 2000; Moran, Kuchner \& Holman 2004). These planets are inferred to be within a few au of the star; however their existence still remains contentious due to the high level of activity of $\epsilon$ Eridani, making interpretation of the spectroscopic measurements difficult (Zechmeister et al. 2013).
The cold outer belt of $\epsilon$ Eridani has been extensively studied from FIR to (sub-)mm wavelengths from the ground (Greaves et al. 1998, 2005; Schütz et al. 2004; Backman et al. 2009; Lestrade \& Thilliez 2015; MacGregor et al. 2015) and from space (Gillett 1986; Backman et al. 2009; Greaves et al. 2014). These observations showed that the debris disc has a ring-like morphology and provided the first estimates of the basic physical properties of the ring such as radial extent, width and inclination. Early sub-millimetre observations conducted with the SCUBA camera on the James Clerk Maxwell Telescope (JCMT; Greaves et al. 1998, 2005) also suggested that the ring has a clumpy structure that has been interpreted as evidence of dynamical interaction between an unseen planetary companion and the debris belt (Quillen \& Thorndike 2002). Substructures in the ring were also identified in Herschel/PACS images at $160 \mu \mathrm{m}$ by Greaves et al. (2014) who, after considering limb brightening effects due to inclination, obtained a 10 per cent flux residual when comparing the flux in the southern portion of the ring to that of the north. Additionally, recent deep (rms $\sim 0.8$ mJy beam $^{-1}$ ) observations made with MAMBO on IRAM at $1.2 \mathrm{~mm}$ (Lestrade \& Thilliez 2015) appear to corroborate the disc inhomogeneities found by SCUBA, though with one of the prominent clumps present in the SCUBA image being absent in the MAMBO map. The time span (18 years since the first sub-mm detection) between the different observational data sets and the large proper motion of $\epsilon$ Eridani, 1 arcsec $\mathrm{yr}^{-1}$, has enabled studies of the outer belt's structure and dynamics, identifying which clumps in the belt could be associated with the disc and which might correspond to background sources, and to look for positional changes of the belt structures over time. The disc orbital motion has been estimated to be of the order of $1^{\circ} \mathrm{yr}^{-1}$ (Greaves et al. 2005) or three times as large (Poulton, Greaves \& Collier Cameron 2006). There are, however, contrasting results. Observations at $1.2 \mathrm{~mm}$ with the bolometer array SIMBA on the SEST telescope (at a depth of $2.2 \mathrm{mJy}^{\text {beam }}{ }^{-1}$ rms) did not confirm the presence of substructure in the ring (Schütz et al. 2004), in agreement with the very recent interferometric map at $1.3 \mathrm{~mm}$ collected with the Submillimeter Array (SMA) by MacGregor et al. (2015). They found that a smooth ring model could explain their patchy high-resolution image. There are other examples in which the presence of dust density enhancements based on early observations at long wavelengths has been questioned by more recent high-resolution imagery. Noteworthy is the case of the very prototypical object Vega for which SCUBA observations in the sub-mm revealed bright blobs (Holland et al. 1998), but whose detection was later disputed (e.g. Hughes et al. 2012).

Motivated by the debated presence of structure along the ring around $\epsilon$ Eridani, its potential correlation with an inferred planet orbiting close to the inner edge of the ring, and the possibility of measuring the orbital motion of dust enhancements within the ring, we conducted deep continuum observations at $1.1 \mathrm{~mm}$ with the AzTEC instrument on the Large Millimetre Telescope Alfonso Serrano $^{1}$ (LMT). In Section 2, we describe the LMT observations and reduction techniques. In Section 3, the global observational morphology of the ring is presented. Section 4 is devoted to the detailed modelling of the ring structure. In Section 5, we briefly discuss the spectra of the components of the system. Section 6 provides additional comments on the flux detected at the stellar position, and in Section 7 we briefly comment on the background sources towards the $\epsilon$ Eridani's system. The concluding remarks are given in Section 8.

\footnotetext{
${ }^{1}$ www.lmtgtm.org
} 


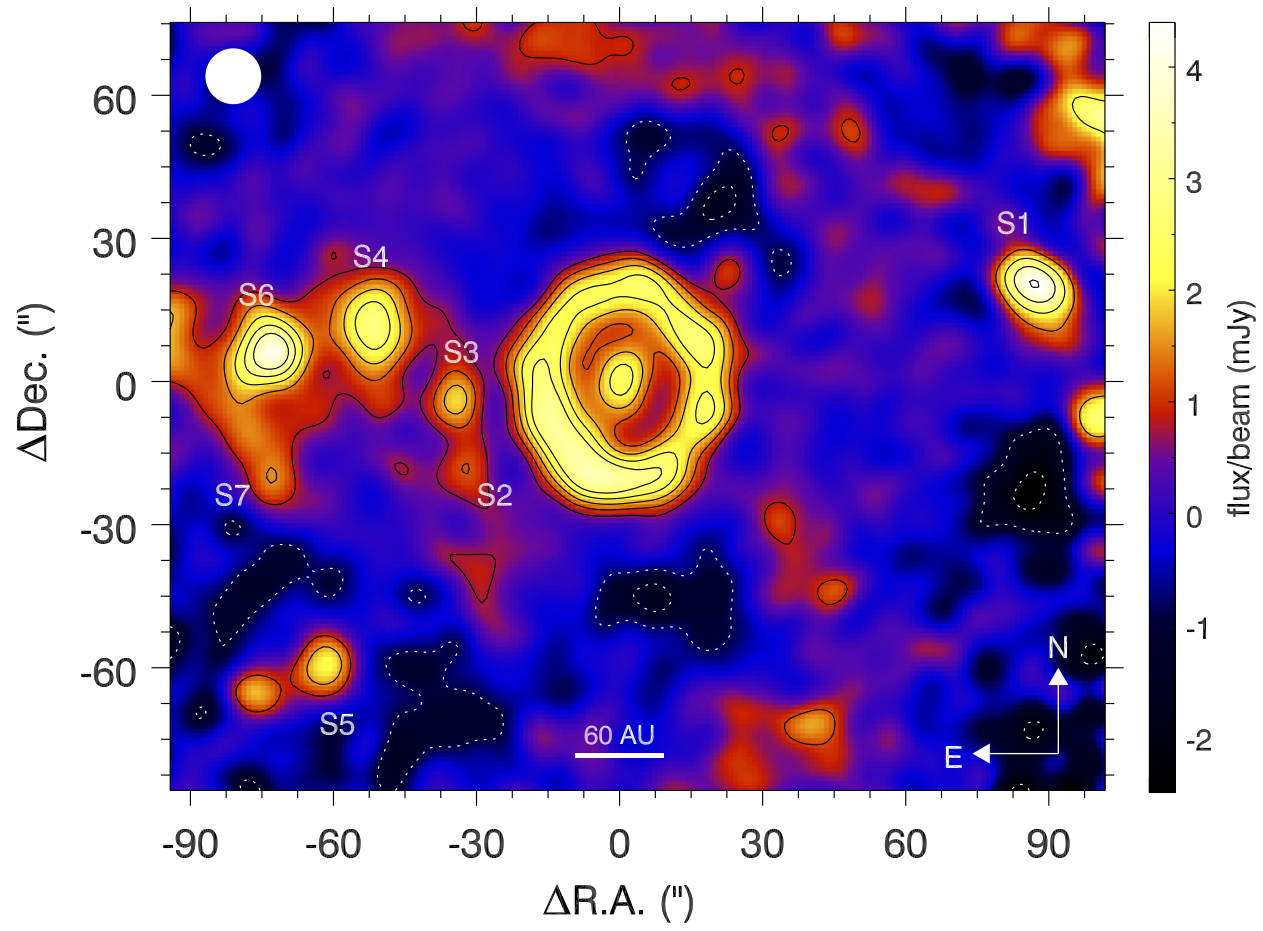

Figure 1. $1.1 \mathrm{~mm}$ LMT/AzTEC continuum map of $\epsilon$ Eridani. The outer ring is fully detected at a significance of $>5.7 \sigma$. The central peak is detected at $7.5 \sigma$ and likely corresponds to the sum of three contributing agents: the stellar photosphere, the stellar upper atmosphere and an (or perhaps two) inner warm disc(s). As many as seven (S1-S7) background objects are detected in this map, of which four have $\mathrm{S} / \mathrm{N}>5$. The source labelled $\mathrm{S} 1$ is the brightest with a $1.1 \mathrm{~mm}$ flux of $4.6 \pm 0.6 \mathrm{mJy}$. The smoothed beam size of 10.9 arcsec FWHM is given by the white circle in the top-left. As a reference, we include the contour levels for $\mathrm{S} / \mathrm{N}=-3.5,-2,2,3.5,5.0,6.5,8.0$.

\section{OBSERVATIONS AND DATA REDUCTION}

Observations with the $1.1 \mathrm{~mm}$ continuum camera AzTEC (Wilson et al. 2008) were conducted in 2014 November and December as part of the Early Science Phase-3 of the LMT, while the 50-m diameter telescope was operating in its $32-\mathrm{m}$ aperture configuration. The telescope is located on top of the extinct volcano Sierra Negra, in the state of Puebla, Mexico, at an altitude of $4600 \mathrm{~m}$ above sea level. A total of $18.5 \mathrm{~h}$ on source were devoted to the target under weather conditions that ranged from excellent to good $\left(\tau_{225 \mathrm{GHz}}=0.03-\right.$ 0.11). The field was observed with the AzTEC small-map observing mode which covers an area of about $7.5 \mathrm{arcmin}^{2}$. The point spread function (PSF) of the instrument in this configuration has a full width at half-maximum (FWHM) beam size of 8.5 arcsec; however, filtering in the reduction process results in an effective resolution of 10.9 arcsec. We made observations of the quasar $0339-017$ roughly every hour which bracketed our observations of the $\epsilon$ Eridani field. The measured quasar pointing offsets (typically $<5$ arcsec) were then interpolated in time to remove any offset and drift in pointing from the science observations.

The raw data were reduced using the standard AzTEC analysis pipeline and analysis approach (Scott et al. 2008; Wilson et al. 2008) but without applying the final Wiener filter that many AzTEC observations use for the optimal detection of point sources. Instead, the final unfiltered image was smoothed with a Gaussian filter with FWHM $=6.8$ arcsec. The data were flux calibrated based on observations of the protoplanetary nebula CRL618 and the noise in the final image is estimated from jackknifed time streams created as described in Scott et al. (2008).
Fig. 1 displays the final AzTEC $1.1 \mathrm{~mm}$ map which has an rms of $0.20 \mathrm{mJy}$ beam $^{-1}$, about 10 times deeper than the SIMBA/SEST observations and four times deeper than those of MAMBO/IRAM. The scale of the map is 1 arcsec pixel ${ }^{-1}$ which results in $93 \operatorname{arcsec}^{2}$ beam $^{-1}$ for the smoothed beam size of 10.9 arcsec. This superb depth reveals three main interesting features in the map: a well-defined complete ring detected for the first time at millimetre wavelengths, a clearly detected central peak, and numerous unresolved background sources, some of which were present in previous maps, in particular those from SCUBA/JCMT and Herschel/PACS and SPIRE instruments (Griffin et al. 2010; Poglitsch et al. 2010). Below, we describe in more detail these characteristics.

\section{MORPHOLOGY OF THE DUSTY RING}

The ring is detected (at a significance ranging from 5.7 to $10.4 \sigma$ ) at all position angles and displays an almost perfect elliptical shape oriented along a north-south direction. Fitting an elliptical ring to the image results in a major axis of 20.0 arcsec (or $64 \mathrm{au}$ ) and the minor axis of $16.9 \operatorname{arcsec}(54 \mathrm{au}$ ), which implies an inclination for a circular ring of about $32^{\circ}$. This latter value is in agreement with the same $32^{\circ}$ derived from Herschel data at $160 \mu \mathrm{m}$ (Greaves et al. 2014) and also, within their uncertainties, with the $\sim 17( \pm 14.2)^{\circ}$ inclination estimated from the SMA map (MacGregor et al. 2015). The centroid of the ring matches the stellar position to within the expected pointing uncertainties of the LMT; therefore we conclude that no offset is detected between the ring and $\epsilon$ Eridani.

The fact that we detect the full ring, clearly separated from a central peak, allows us to provide more observational constraints 


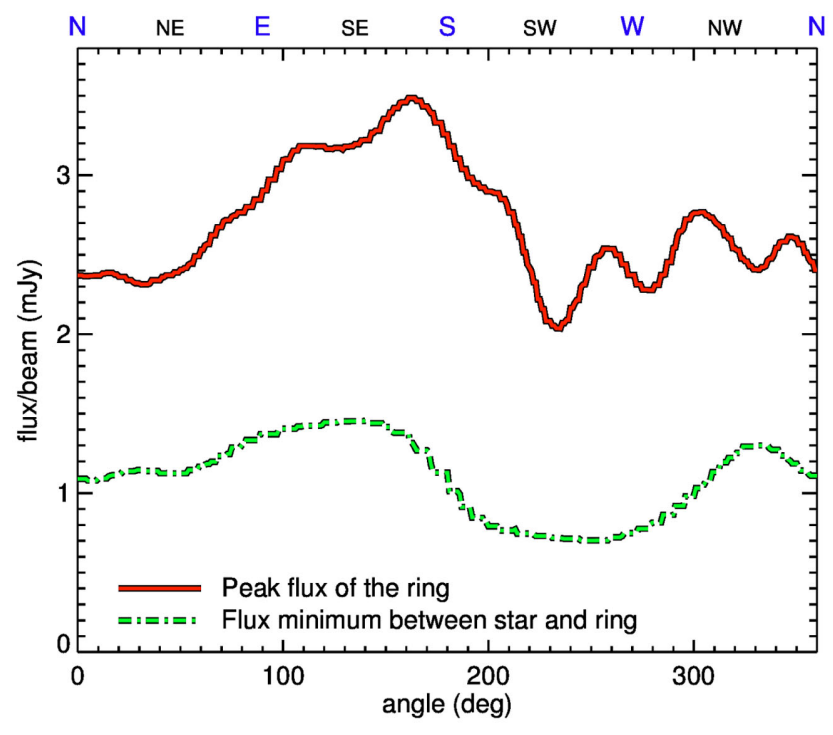

Figure 2. Azimuthal flux distribution showing the variations of the flux maxima along the ring (continuous line). On average, the ring flux has a level of $2.4 \mathrm{mJy}_{\text {beam }}{ }^{-1}$, and the brightest arc of the ring is in the SE as can be seen in the map of Fig. 1. The flux peak in this bright arc is $\sim 3.5 \mathrm{mJy}$ at about $160^{\circ}$, measured counter-clockwise from north. The green dot-dashed line indicates the flux distribution in the gap which is also fully detected. The SE-NW ansae seen in Fig. 1 corresponds to the bumps in the gap distribution. The origin of this brightening is not known, but can plausibly be a reduction artefact.

on the basic properties needed for the disc modelling, namely: the distance from the star (the ring radius $R$ ), the ring width (upper limit), and the dust properties obtained through the integrated flux density. In unresolved discs, whose dust characteristics can only be inferred through the analysis of the SED, some of the above derived properties are, as mentioned before, degenerate.

In addition to the ring radius $R$ and the inclination mentioned above, the ring appears unresolved in the radial direction (width $<11$ arcsec or $\sim 35 \mathrm{au}$ ) and has an integrated flux density, obtained through standard aperture photometry analysis, of $27.7 \pm 3.3 \mathrm{mJy}$, compatible with the values obtained at millimetre wavelengths with IRAM/MAMBO (Lestrade \& Thilliez 2015) and the SMA (MacGregor et al. 2015).

Morphologically, the ring in the LMT map shows a prominent extended brightening in the SE and three apparent clumps in the NW. This SE flux enhancement is evident from the azimuthal flux peak distribution depicted with the red line of Fig. 2. The maximum is at $\sim 160^{\circ}$ from the north counterclockwise reaching about 3.5 mJy beam ${ }^{-1}$, which is $\sim 50$ per cent above the lowest flux levels of the ring in the $0-40^{\circ}$ and $230^{\circ}-360^{\circ}$ segments. This flux difference is certainly less notable than the factor of 3 reported in Greaves et al. (2005, their fig. 3) and the factor of 4 in Lestrade \& Thilliez (2015). This SE bright arc is neither seen in the MAMBO map nor in SMA data. Conversely, the apparent clump in the SW in the SCUBA and MAMBO maps is not seen in the LMT image. While there are discrepancies, it is important to note that the published SCUBA data provided the most complete ring maps in the imaging data collected prior to these LMT observations, and that the bright arc in the SE has been regarded as a real feature of the ring. Whilst a direct inspection of the positions of the flux maxima of this SE arc in the two SCUBA and the LMT images appears to indicate motion

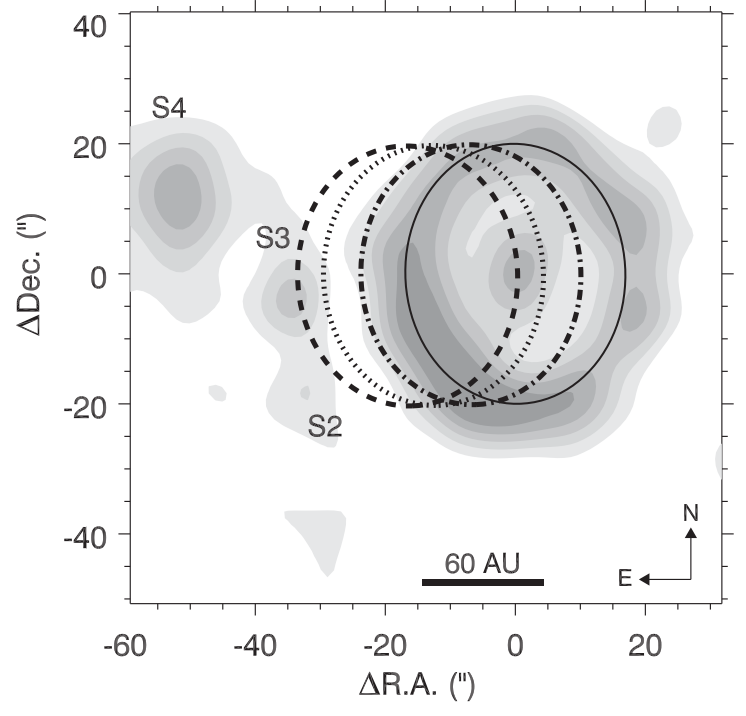

Figure 3. Zoomed version of the $1.1 \mathrm{~mm}$ LMT/AzTEC continuum map of $\epsilon$ Eridani. The map is presented in grey-scales that correspond to the contour levels of Fig. 1. The ellipses denote the ring positions at different epochs with respect to background point sources due to the proper motion of the system. The ellipses with thin solid, dot-dashed, dotted, and dashed lines show the location of the $\epsilon$ Eridani system at the epochs of the observations with AzTEC (2014.9), MAMBO (2007.9), and SCUBA (2002.0, 1997.9), respectively. Note that the source $\mathrm{S} 3$ almost coincides with the ring position at the first SCUBA observations of Greaves et al. (1998).

of substructures, the two sources east of the ring in the LMT map, in particular that labelled S3 in Fig. 1, could conceivably explain the apparent flux enhancement in the SCUBA map of Greaves et al. (1998) as its position agrees with that of the ring at the time when the SCUBA observations were carried out. Similarly, the suggested background sources as the origin of the flux brightening in the SW in the first SCUBA map could now well partially contribute to the LMT SE brightening. In Fig. 3 we show the positions of the dust ring with respect to the stationary background sources at different epochs correspondent to previous (sub-)mm observations.

To further verify the presence (or lack) of substructures along the ring, we modelled the $\epsilon$ Eridani LMT image using optically thin debris disc models as presented below.

\section{$4 \epsilon$ ERIDANI MODELLING}

We modelled the emission from $\epsilon$ Eridani using a parametrized model of an optically thin debris disc. This model has been described in detail in Wyatt et al. (1999) and Kennedy et al. (2012). Basically, a 3D distribution of dust surface area is generated, which can be viewed from any direction to create synthetic images. The surface brightness of the images is calculated by adding up the emission in individual cells along the line of sight, where each cell emits like a blackbody at some temperature that is proportional to the distance to the central star. For the resolved disc components we used a temperature law of $T_{\text {res }}=416 / \sqrt{R} \mathrm{~K}$, where $R$ is the disc radius in au. Though we model the data at a single wavelength, this temperature was chosen to provide a reasonable extrapolation of the model to the photometric data at other wavelengths. For debris discs this model can account for important effects such as brightening at disc ansae and brightness asymmetry for non-axisymmetric discs. 

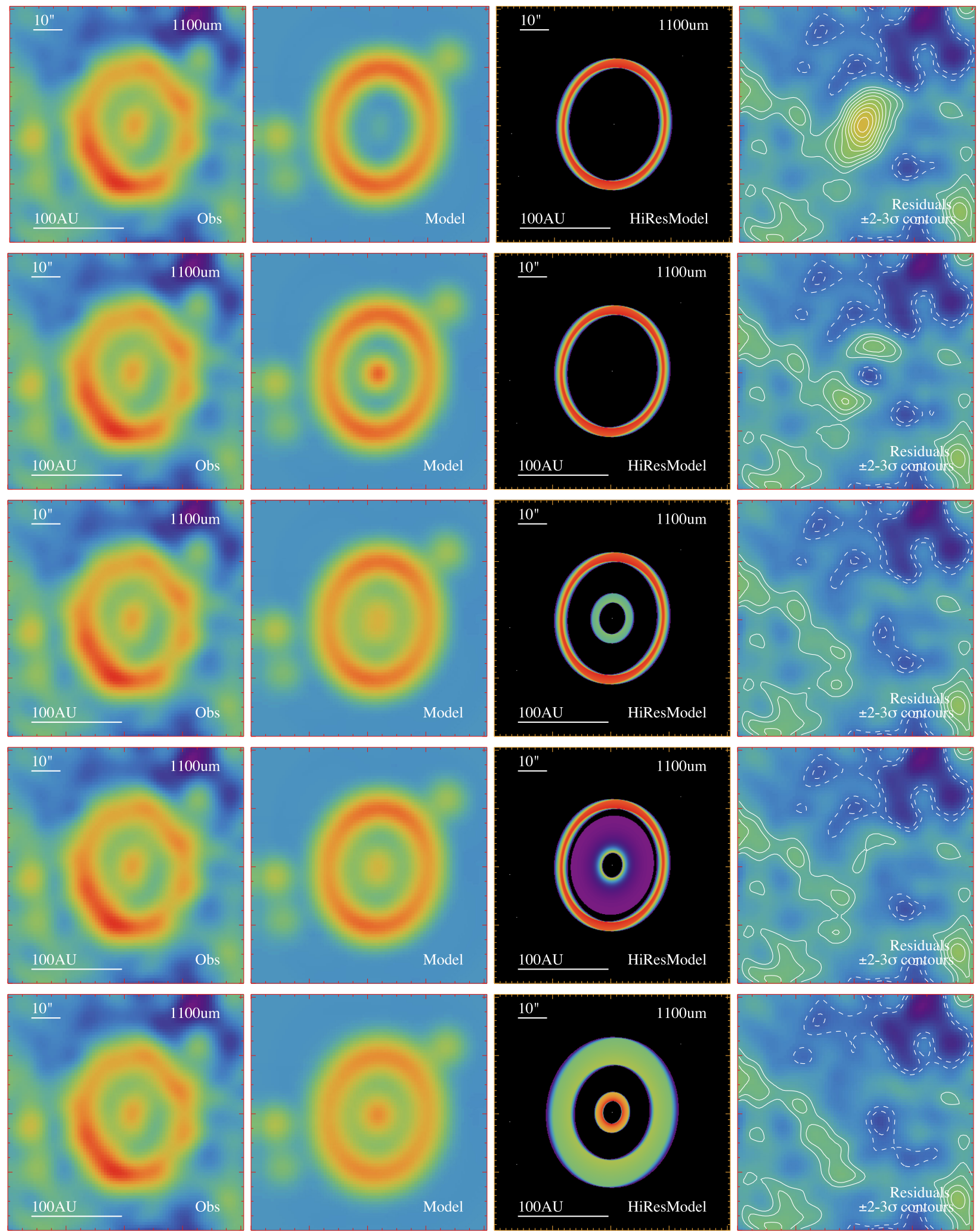

Figure 4. Models of the LMT $1.1 \mathrm{~mm}$ image of $\epsilon$ Eridani. Each row is a different model, with the panels showing, from left to right, the data, the model at the resolution of the 32-m LMT, the high-resolution model, and the residuals. The residuals include contours in units of S/N from $\pm 2 \sigma$. The panels of the first and second rows show an outer ring centred at 68 and 10 au wide, with the stellar photosphere ( $0.7 \mathrm{mJy}$ ) as the only contributor in the inner region (first row), and an artificial point source of $2.7 \mathrm{mJy}$ added at the stellar position (second row). The third row considers an outer ring as in the above panels and includes an inner ring 10 au wide at 23 au. In the fourth row the inner component is wider, extending from 14 to 63 au, but follows a surface density power law with index $=-3.5$ in order to keep it concentrated. In the bottom row panels we considered a wider ( $30 \mathrm{au}$ ) outer ring centred at 69 au, with a narrow (10 au) inner component at 18 au. 
For a given model viewed at some orientation, a high-resolution disc image is first generated. The central stellar emission of $0.7 \mathrm{mJy}$ (i.e. the expected photospheric flux; see the next section) is then added and the model is convolved with the LMT beam. A $\chi^{2}$ goodness of fit metric is then computed within an $80 \times 80 \operatorname{arcsec}^{2}$ area shown in Fig. 4, but only pixels (about 70 per cent) where the emission from either the model or the image is significant are used (for details see Wyatt et al. 2012). The variable background level means that simple least-squares minimization does not necessarily yield satisfactory results, so in most cases some by-hand intervention was needed to obtain a smooth and continuous background. That is, even though models with $\chi^{2}$ values lower than those presented are possible, they remove what could be astrophysical background behind the disc, in particular at the SE side, and produce negative residuals in the NW. While the $\chi^{2}$ is always several times higher than the number of degrees of freedom (because our model of the sky near $\epsilon$ Eri is incomplete), visually better fitting models do have lower $\chi^{2}$ values. As is commonly the case with models of low spatial-resolution data, we do not explore all possible parameter space and so do not claim that our models are unique, but that they are reasonable interpretations of the LMT data.

The outer ring at $\sim 70$ au is clear in the LMT image, so the main goals of the modelling were (i) to determine whether the ring width was well constrained, (ii) determine whether additional emission above that expected from the star is present interior to the outer ring, and (iii) look for any evidence of azimuthal structure.

Additional issues for this modelling were the overall flux calibration and any flux (DC) offset, and nearby background sources. We allowed for a small DC offset in modelling the images, though it did not influence the results. Near the disc three background sources were added, one corresponding to the blob in the NW, and two to the E and SE sources labelled S3 and S2 in Fig. 1, respectively. As we note below, there is the possibility of additional emission that exists behind the SE portion of the disc, which could plausibly be an extension of the diffuse emission to the $E$ of the system.

\subsection{Ring width}

To address point (i) above, we constructed models with a range of ring widths with constant surface density centred at a distance of about 70 au from the star. These models consider an inclination of $30^{\circ}$ which, unlike previous observational results at millimetre wavelengths (e.g. MacGregor et al. 2015), is strongly constrained. The position angle is $7^{\circ}$ from north to west (i.e. slightly west of north), so the geometry is consistent with previous results (Greaves et al. 2014; MacGregor et al. 2015). Models with arbitrarily narrow ring widths reproduced the data reasonably well, with $\chi_{\text {red }}^{2}$ values of around 4.7 (where $\chi_{\text {red }}^{2}$ is the reduced $\chi^{2}$ ), and these models also have a good fit to the dust emission interior to the main ring. We also constructed models with wide rings, finding that a width of up to 30 au was acceptable, but for larger sizes the ring surface brightness becomes too low, i.e. for a 30 au width $\chi_{\text {red }}^{2}=5$. Some example models are shown in Fig. 4. The lower two rows of panels show the results for narrow (10 au) and wide (30 au) outer belts, both of which produce reasonable results. Thus, we conclude that the LMT image does not place a lower limit on the ring width and that it could be as wide as $30 \mathrm{au}$, conclusions that compare well with those of Lestrade \& Thilliez (2015) and MacGregor et al. (2015).

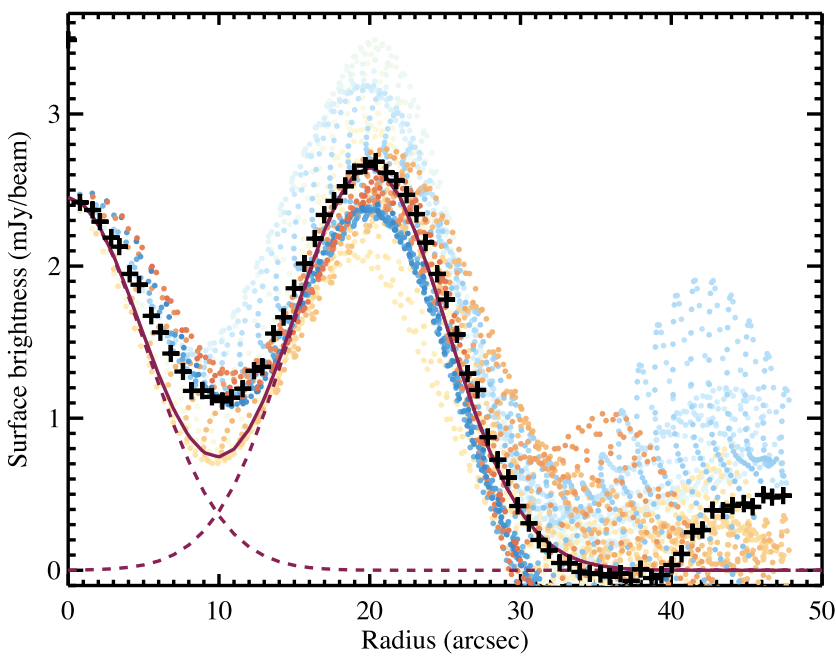

Figure 5. Radial flux distribution of the $\epsilon$ Eridani system. The dotted lines are the Gaussian profiles fitted to the central peak and the ring. They both match the PSF of 11 arcsec, hence not resolved. The continuous line is the sum of these profiles. Coloured dots represent the full set of flux points in each azimuthal direction, and the average observed fluxes are depicted with the ' + ' symbols. Note that significant diffuse flux is present in the gap at 10 arcsec radius.

\subsection{Non-stellar emission interior to the ring}

To address the second issue, we constructed models with an additional interior component. The inclusion of this extra component is motivated by two facts: (1) we expect a contribution of an excess above the photosphere from the star originating from the warm belt(s) and (2) the significant flux in the gap between the star and the ring we found in the LMT map (green dot-dashed line in Fig. 2).

The width and brightness of this component were varied to ascertain whether its existence was required by the data, and if so, the extent of this component. The above models that include only the outer belt leave significant residuals in the interior regions $\left(\chi_{\text {red }}^{2}=8.2\right)$, so we conclude that mm-wave emission exists in excess of the stellar photospheric emission interior to the outer belt (e.g. top row of panels in Fig. 4). A $2.7 \mathrm{mJy}$ (i.e. $2 \mathrm{mJy}$ above the photosphere) point source at the stellar position produces marginally satisfactory results $\left(\chi_{\text {red }}^{2}=5.3\right)$, but emission remains between the star and the outer belt (second row of panels). The same conclusion can be reached by considering the radial flux distribution shown in Fig. 5, where the outer belt appears unresolved but the interior flux cannot be explained by simply adding a point source at the star position. This flux is somewhat overestimated, as can be seen by the negative residuals at the star position. We therefore favour models with extended emission between the star and the outer belt. Models with interior emission included can reproduce the data fairly easily; both continuous emission from a flat surface density disc extending from 14 to 63 au and a narrow belt at 30 au produced satisfactory results $\left(\chi_{\text {red }}^{2} \approx 4.7\right.$; see the third and fourth rows in Fig. 4). Thus, while we are confident that the interior emission exists, we cannot constrain from where this emission originates. In most models a small amount of emission was required at the stellar position, but the level is degenerate with the structure of the emission between the star and the outer belt. 


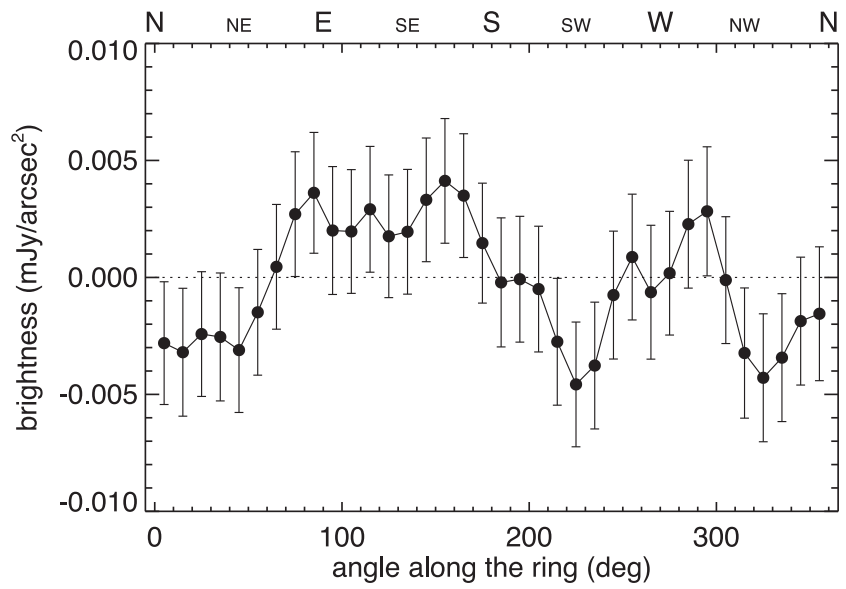

Figure 6. Flux residual obtained by subtracting a well-fitting model flux to that of the observed map. The residual indicates that, to within the uncertainties, the ring has a smooth morphology. A reduced $\chi^{2}$ of the data points results in 0.92 , supporting lack of substructures.

\subsection{Azimuthal structure}

In all cases where a reasonable fit to the data was obtained there was no evidence for significant non-axisymmetric emission in the residuals. In most cases emission remained in the SE part of the ring, but, given the extended structure seen to the $\mathrm{E}$ of the system, attributing this residual emission to the $\epsilon$ Eridani system is not well justified.

Complementarily, we carried out a similar analysis as that of MacGregor et al. (2015), namely, we calculated the residual emission by subtracting a best-fitting model of the ring to the integral of the observational flux calculated in sectors with a central angle of $10^{\circ}$ extending from 10 to 28 arcsec from the centre. Note that the outer radius of the annulus is slightly smaller than in MacGregor et al. (2015) because we wanted to avoid contamination from the faint NW source close to the ring. The azimuthal distribution of the residual flux is displayed in Fig. 6. Note that the only potential feature detected in the NE quadrant of the SMA map is not present in our map. To within the uncertainties, the distribution shown in this figure indicates that the ring has a smooth structure, with perhaps the presence of two regions of low flux at azimuthal angles of $\sim 220^{\circ}$ and $\sim 320^{\circ}$.

The various components in the best-fitting models have a $1.1 \mathrm{~mm}$ flux of $\sim 25 \mathrm{mJy}$ for the outer ring, in good agreement with the photometric result shown above, and a total of $5.5 \mathrm{mJy}$ for the interior regions. A flux of $0.7 \mathrm{mJy}$ is attributed to the stellar photosphere. For the model where the interior disc component is a narrow ring the flux is $3.4 \mathrm{mJy}$, and when it is extended the flux is $4.5 \mathrm{mJy}$.

\section{THE SPECTRUM OF THE $\epsilon$ ERIDANI SYSTEM}

The infrared to millimetre spectrum of the $\epsilon$ Eridani system is depicted in Fig. 7. Three separate components are shown in this figure. The solid brown line represents the photosphere that was generated from an interpolation within the library of synthetic spectra of Castelli \& Kurucz (2003). These synthetic fluxes are calculated up to $160 \mu \mathrm{m}$, so an extrapolation of the Rayleigh-Jeans tail was necessary to account for the flux at longer wavelengths. We have assumed the stellar parameters derived by Paletou et al. (2015):

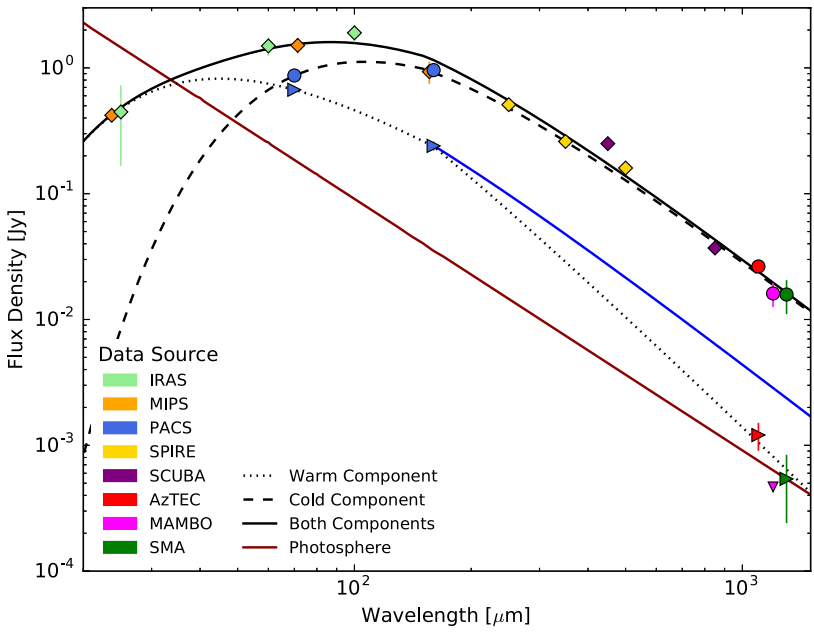

Figure 7. Spectrum of $\epsilon$ Eridani. Different lines styles represent the different components of the system: the photosphere (solid red), the outer belt (dashed), the inner component (dotted), and the summed contributions (thick solid). The instrumental origin of the ancillary data is indicated in the figure inset and the symbols stand for data that contain fluxes for the inner component (triangles), the outer belt (circles), and those of the whole system (diamonds). These data have been partially compiled by Greaves et al. (2014) from Backman et al. (2009) and Greaves et al. (2005). The outer belt spectrum has been constructed with the parameters $T=48 \mathrm{~K}, \lambda_{0}=$ $150 \mu \mathrm{m}$, and $\beta=0.4$, as in Greaves et al. (2014). The inner component, instead, represents the best fit of the four available points, two from Herschel PACS at 70 and $160 \mu \mathrm{m}$, the SMA data point at $1.3 \mathrm{~mm}$, and the LMT/AzTEC flux at $1.1 \mathrm{~mm}$. The best-fitting parameters assuming $\lambda_{0}=$ $150 \mu \mathrm{m}$ are $T=113 \mathrm{~K}$ and $\beta=1.0$. The solid blue line shows the long wavelength extension of the grey body emission for the inner component considering an emissivity index of $\beta=0.4$. The two data points at long wavelengths (and the upper limit of MAMBO at $1.2 \mathrm{~mm}$ ) provide meaningful constraints on the dust properties of the warm belt.

$\left(T_{\text {eff }} / \log g /[\mathrm{Fe} / \mathrm{H}]\right)=(5034 / 4.51 /-0.12)$. The dashed curve indicates the location of the modified blackbody curve for a temperature of $48 \mathrm{~K}, \lambda_{0}=150 \mu \mathrm{m}$, and an emissivity index $\beta=0.4$ (Greaves et al. 2014). Ancillary data (see labels in the figure) are also plotted for illustrative purposes and no formal fit of the points was attempted. The data, in spite of some dispersion, are well represented by the above modified blackbody parameters. The dispersion can be partially attributable to the differing components that are included in the integrated flux densities calculated from distinct data sets. The dotted curve displays the best fit provided by the Herschel, SMA and LMT data for the inner component. The latter data point (1.3 mJy) includes only the unresolved central emission after subtracting the photospheric (and chromospheric; see the next section) contribution considering $\lambda_{0}=150 \mu \mathrm{m}$. The resulting best-fitting parameters of the warm component are $T=113 \mathrm{~K}$ and $\beta=1.0$. The blue line shows the extension of the modified blackbody for the inner component using the parameters of Greaves et al. (2014). The solid thick black curve corresponds to the summed contributions of the photosphere, the inner flux peak, and outer belt.

With the available data, dust masses $\left(M_{\text {dust }}\right)$ can be calculated for the different components. As in Greaves et al. (1998), we consider two cases for the absorption coefficient; $k_{850 \mu \mathrm{m}}=$ 1.7 and $0.4 \mathrm{~cm}^{2} \mathrm{gr}^{-1}$ which at $1.1 \mathrm{~mm}$ correspond to $k_{1.1 \mathrm{~mm}}=$ $(850 / 1100)^{\beta} \times k_{850 \mu \mathrm{m}} \mathrm{cm}^{2} \mathrm{~g}^{-1}$. At a distance of $3.22 \mathrm{pc}, T=48 \mathrm{~K}$, and $\beta=0.4\left(k_{1.1 \mathrm{~mm}}=1.53\right.$ and $\left.0.36 \mathrm{~cm}^{2} \mathrm{~g}^{-1}\right), M_{\text {dust }}$ for the cold 
outer belt is 0.0035 and $0.015 \mathrm{M}_{\oplus}$, which are slightly smaller, but compatible with those reported by Greaves et al. (1998).

For the inner component we conducted two calculations: (a) we first considered the best-fitting parameters obtained above for the unresolved warm component and the transformed absorption coefficients $k_{1.1 \mathrm{~mm}}=1.31$ and $0.31 \mathrm{~cm}^{2} \mathrm{~g}^{-1}$ for $\beta=1.0$. The resulting $M_{\text {dust }}$ are $7.6 \times 10^{-5}$ and $0.0003 \mathrm{M}_{\oplus}$ for the two coefficient values, respectively; (b) we assumed an inner component that includes both the unresolved component and a narrow warm disc with a total flux of $3.4 \mathrm{mJy}$, in agreement with an emissivity index of $\beta=0.4$. In this case the dust mass is $M_{\text {dust }}=0.00017$ and $0.0007 \mathrm{M}_{\oplus}$, for the high and low values of $k_{1.1 \mathrm{~mm}}$, respectively. According to these results there is a similar amount of warm dust $\left(0.0001 \mathrm{M}_{\oplus}\right)$ very close to the star and in between the central unresolved emission and the external cold belt which could be, as mentioned before, evidence of material being transported to the inner regions from the outer relatively massive debris ring.

\section{ADDITIONAL COMMENTS ON THE CENTRAL PEAK}

The unresolved emission from the central peak is detected at $7.5 \sigma$. The integrated flux in the centre is $2.3 \pm 0.3 \mathrm{mJy}$ and arises from several potential contributors, namely, the stellar atmosphere (photosphere and chromosphere) and the Rayleigh-Jeans tail of the warm dust component(s) identified at 70 and $160 \mu \mathrm{m}$ with Herschel/PACS (Greaves et al. 2014). As mentioned above, the expected photospheric contribution to the total flux at $1.1 \mathrm{~mm}$ is $0.7 \mathrm{mJy}$, implying a potential warm dust contribution of $\sim 1.6 \mathrm{mJy}$. The blue line in Fig. 7 predicts an excess of $\sim 3.5 \mathrm{mJy}$ at $1.1 \mathrm{~mm}$, which is nearly twice as much as that measured in our LMT map. In fact, the addition of the two extra data points provided by the LMT and the SMA results in a much steeper $\beta$ index and suggests that $\lambda_{0}$ should remain close to the Herschel/PACS band at $160 \mu \mathrm{m}$. In addition, it has been found that active stars present a non-monotonic temperature variation towards the upper atmospheric layers, in a similar way the Sun displays a temperature increase towards low optical depths after reaching a minimum temperature in the atmosphere at about $\log \tau=-4$. This temperature gradient reversal is commonly evidenced by an excess in the ultraviolet regime but also as an atmospheric (sub-)mm-wave excess. In a recent study, Liseau et al. (2015) found that $\alpha$ Cen B (K1 V) displays an excess at bands 9, 7 and $3(440 \mu \mathrm{m}, 870 \mu \mathrm{m}$ and $3 \mathrm{~mm}$, respectively) of the Atacama Large Millimetre/submillimetre Array (ALMA). Considering that $\alpha$ Cen B and $\epsilon$ Eridani have similar spectral types and assuming that both stars have a similar degree of activity, as suggested by their far and mid-ultraviolet (1200-2500 ̊) continuum flux surplus, it is not unreasonable to expect a similar excess in $\epsilon$ Eridani. The comparison of observed and predicted fluxes of $\alpha$ Cen B indicates that in ALMA bands 7 and 3 the observed stellar fluxes are, respectively, 40 and 220 per cent higher than predicted for the photosphere. Based on observations conducted with the Australia Telescope Compact Array (ATCA) at $7 \mathrm{~mm}$, MacGregor et al. (2015) reported a flux excess of about a factor of 3 , which agrees with the trend of an increasing excess at longer wavelengths. These authors argue that no plausible inner disc scenario is able to explain the observed excess and ascribe it to a thermal origin in the upper atmosphere of $\epsilon$ Eridani. An interpolation of the bracketing ALMA points to the $1.1 \mathrm{~mm}$ band results in an excess of $\sim 50$ per cent, which would imply a total stellar flux of $\sim 1 \mathrm{mJy}$ for $\epsilon$ Eridani. This atmospheric value actually leaves $1.3 \mathrm{mJy}$ at $1.1 \mathrm{~mm}$ originating from the unresolved warm belt(s) and, therefore, its contribution would be about
Table 1. Positions and flux densities of background sources.

\begin{tabular}{lrrr}
\hline Source & $\begin{array}{c}\text { Offset RA } \\
(\operatorname{arcsec})\end{array}$ & $\begin{array}{c}\text { Offset Dec. } \\
(\operatorname{arcsec})\end{array}$ & $\begin{array}{c}\text { Flux } \\
\left(\mathrm{mJy} \mathrm{beam}^{-1}\right)\end{array}$ \\
\hline Central peak & $\ldots$ & $\ldots$ & $2.3 \pm 0.3$ \\
S1 & 85.9 & 20.0 & $4.6 \pm 0.6$ \\
S2 & -32.5 & -18.7 & $1.2 \pm 0.3$ \\
S3 & -34.5 & -4.4 & $1.9 \pm 0.3$ \\
S4 & -52.0 & 11.4 & $3.0 \pm 0.3$ \\
S5 & -62.0 & -59.7 & $2.3 \pm 0.4$ \\
S6 & -73.1 & 5.6 & $4.2 \pm 0.4$ \\
S7 & -73.1 & -20.0 & $1.5 \pm 0.3$ \\
\hline
\end{tabular}

Note. Offsets are given with respect to the stellar position. At the time of the observations (2014.9) $\epsilon$ Eridani coordinates were $\alpha=03: 40: 13.1$ and $\delta=-09: 24: 38.6$, which include a correction for proper motion of

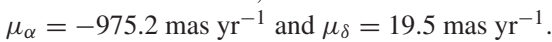

half of the measured central emission. Complementing the LMT and SMA data with measurements of the central source at other (sub-)mm bands will be very valuable to better assess the appropriate contributions of the sources of millimetre emission. These contributions will allow us to better constrain the dust properties of the warm belt and to understand the outer atmospheric structure of $\epsilon$ Eridani.

\section{BACKGROUND SOURCES}

As mentioned before, another important feature of our map is that, thanks to the depth and enhanced resolution achieved, we found numerous sources whose nature has been suggested, but never been previously investigated. In addition to the cold ring and the central peak, seven point sources were detected with a significance $\geq 3.5 \sigma$ and fluxes that range from 1.2 to $4.6 \mathrm{mJy}$ (see Table 1). These sources do not have near- or mid-IR counterparts on either 2MASS or WISE images, but some are also present in previous Spitzer (Rieke et al. 2004, MIPS-70 $\mu \mathrm{m}$ ), Herschel (PACS and SPIRE at 70, 160, 250, 350 and $500 \mu \mathrm{m}$ ), and, particularly, in SCUBA and SCUBA2 images at 450 and $850 \mu \mathrm{m}$. For several of the sources (S2, S3, and S7) our map provides the first detection. It has been suggested by Greaves et al. $(1998,2005)$ that these sources are probably members of the population of distant and heavily obscured submillimetre galaxies (SMG; Smail, Ivison \& Blain 1997; Hughes et al. 1998). Considering the area of the map, we expect $\sim 10$ SMGs to be present in our map for a detection threshold of $S_{1.1 \mathrm{~mm}}=0.7 \mathrm{mJy}$ (Scott et al. 2012; Shimizu, Yoshida \& Okamoto 2012), which is consistent with the number of point sources detected in the AzTEC map. It also agrees with the number counts derived in the very recent study of Fujimoto et al. (2016) scaled to the $1.1 \mathrm{~mm}$ wavelength for a spectral index of 3 . The analysis of these background sources is beyond the scope of this paper. We, nevertheless, would like to remark that photometric and molecular line studies are required to reveal the (distant or nearby) nature of the sources surrounding the high Galactic latitude $\left(|\mathrm{b}|=48^{\circ}\right)$ target $\epsilon$ Eridani.

\section{SUMMARY AND CONCLUSIONS}

We present the deepest $(0.2 \mathrm{mJy} \mathrm{rms})$ single-dish observations at millimetre wavelengths of the prototypical debris disc target $\epsilon$ Eridani conducted with the AzTEC camera on the LMT. Our $7.5 \operatorname{arcmin}^{2}$ image reveals the stellar emission, the cool disc, and nearby (line-of-sight) environment with the following features. 
(i) The ring is detected for the first time at all position angles. The ring has a measured radius of $20 \operatorname{arcsec}$ or $64 \mathrm{au}$, and an upper limit of the width of 30 au derived from model fitting, which implies a relative ring width $(\Delta R / R)$ of $\leq 0.5$. These values are in agreement with previous observational and modelled properties. The ring shows some inhomogeneities that could be explained by the presence of background objects that coincide with the ring position. Bright structures in the ring previously observed at comparable submm wavelengths with SCUBA can also be ascribed to background objects, currently separated from the ring due to the star's high proper motion. Modelling of the ring indicates that its morphology is essentially smooth, and that a potential residual brightening in the SE might be an extension of the diffuse emission east from the system.

(ii) The central peak, which includes a stellar contribution and one or perhaps two warm dust belts, is also clearly detected. These LMT observations, along with the recent SMA data and archival Herschel fluxes at 70 and $160 \mu \mathrm{m}$, where the resolution is also good enough to separate the outer ring and the central peak, indicate that the interior warm dust contributes approximately 60 per cent of this emission. The theoretical analysis of both the central peak and the outer dust ring shows evidence of significant emission in the gap. This may constitute the first evidence of the Pointing-Robertson drag outside the Solar system.

(iii) Numerous point sources are detected around the $\epsilon$ Eridani system. The sources most likely correspond to a population of massive distant star-forming galaxies as has been suggested in previous works. Further analyses are needed to verify the nature and the properties of their cool dust component.

Our $1.1 \mathrm{~mm}$ observations demonstrate the current capabilities of the operational LMT in the study of nearby circumstellar debris discs. We have traced the full extent of the nearest debris disc/Edgeworth-Kuiper belt analogue to the Solar system for the first time. At the same time we have identified a number of line-ofsight background sources, which could be members of the sub-mm bright, high-redshift population of star-forming galaxies. Disentangling these extragalactic sources from excesses around disc-host stars is critical for the proper interpretation of planetary systems analogous to our own. The LMT is expected to operate at its full aperture capacity of $50 \mathrm{~m}$ in 2017 . The resolution to be achieved in this final 50-m diameter configuration of the LMT is 5 arcsec, and when combined with the increased sensitivity, stronger constraints on the ring properties will be observationally established.

\section{ACKNOWLEDGEMENTS}

This work would have not been possible without the long-term financial support from the Mexican Science and Technology Funding Agency, CONACyT (Consejo Nacional de Ciencia y Tecnología) during the construction and operational phase of the Large Millimetre Telescope Alfonso Serrano, as well as support from the US National Science Foundation via the University Radio Observatory program, the Instituto Nacional de Astrofísica, Optica y Electrónica (INAOE) and the University of Massachusetts, Amherst (UMass). MC, EB, FCSM, MO and RLV work was supported by CONACyT research grants SEP-2009-134985 and SEP-2011-169554. GMK is supported by the Royal Society as a Royal Society University Research Fellow. CE is partly supported by Spanish grant AYA201455840-P. JPM is supported by a UNSW Vice Chancellor's Postdoctoral Fellowship. SL acknowledges support from CONACyT through grant 238631. We are grateful to all of the LMT person- nel and observers from Mexico and UMass who made this project possible.

\section{REFERENCES}

Augereau J. C., Lagrange A. M., Mouillet D., Papaloizou J. C. B., Grorod P. A., 1999, A\&A, 348, 557

Backman D. E., Paresce F., 1993, in Levy E. H., Lunine J. I., eds, Protostars and Planets III. Univ. Arizona Press, Tucson, AZ, p. 1253

Backman D. et al., 2009, ApJ, 690, 1522

Bonfanti A., Ortolani S., Piotto G., Nascimbeni V., 2015, A\&A, 575, A18

Bryden G. et al., 2013, in American Astronomical Society Meeting Abstracts 221. p. 144.24

Castelli F., Kurucz R. L., 2003, in Piskunov N., Weiss W. W., Gray D. F., eds, IAU Symposium Vol. 210, Modelling of Stellar Atmospheres. Publications of the Astronomical Society of the Pacific, San Francisco, p. A20

Di Folco E., Thévenin F., Kervella P., Domiciano de Souza A., Coudé du Foresto V., Ségransan D., Morel P., 2004, A\&A, 426, 601

Eiroa C. et al., 2013, A\&A, 555, A11

Ertel S. et al., 2014, A\&A, 561, A114

Fujimoto S., Ouchi M., Ono Y., Shibuya T., Ishigaki M., Nagai H., Momose R., 2016, ApJS, 222, 1

Gillett F. C., 1986, in Israel F. P., ed., Astrophysics and Space Science Library Vol. 124, Light on Dark Matter. D. Reidel Publishing Co, Dordrecht, p. 61

Greaves J. S. et al., 1998, ApJ, 506, L133

Greaves J. S. et al., 2005, ApJ, 619, L187

Greaves J. S. et al., 2014, ApJ, 791, L11

Griffin M. J. et al., 2010, A\&A, 518, L3

Hatzes A. P. et al., 2000, ApJ, 544, L145

Holland W. S. et al., 1998, Nature, 392, 788

Hughes D. H. et al., 1998, Nature, 394, 241

Hughes A. M. et al., 2012, ApJ, 750, 82

Kennedy G. M. et al., 2012, MNRAS, 421, 2264

Krivov A. V., 2010, Res. Astron. Astrophys., 10, 383

Krivov A. V., Müller S., Löhne T., Mutschke H., 2008, ApJ, 687, 608

Lagrange A.-M. et al., 2010, Science, 329, 57

Lebreton J. et al., 2012, A\&A, 539, A17

Lestrade J.-F., Thilliez E., 2015, A\&A, 576, A72

Liseau R. et al., 2015, A\&A, 573, L4

MacGregor M. A., Wilner D. J., Andrews S. M., Lestrade J.-F., Maddison S., 2015, ApJ, 809, 47

Maldonado J., Eiroa C., Villaver E., Montesinos B., Mora A., 2012, A\&A, 541, A40

Mamajek E. E., Hillenbrand L. A., 2008, ApJ, 687, 1264

Marshall J. P. et al., 2014a, A\&A, 565, A15

Marshall J. P. et al., 2014b, A\&A, 570, A114

Matthews B. C., Krivov A. V., Wyatt M. C., Bryden G., Eiroa C., 2014, in Beuther H. et al., eds, Protostars and Planets VI. Univ. Arizona Press, Tucson, AZ, p. 521

Morales F. Y., Rieke G. H., Werner M. W., Bryden G., Stapelfeldt K. R., Su K. Y. L., 2011, ApJ, 730, L29

Moran S. M., Kuchner M. J., Holman M. J., 2004, ApJ, 612, 1163

Moro-Martín A. et al., 2015, ApJ, 801, 143

Nilsson R. et al., 2010, A\&A, 518, A40

Paletou F., Böhm T., Watson V., Trouilhet J.-F., 2015, A\&A, 573, A67

Panić O. et al., 2013, MNRAS, 435, 1037

Pawellek N., Krivov A. V., Marshall J. P., Montesinos B., Ábrahám P., Moór A., Bryden G., Eiroa C., 2014, ApJ, 792, 65

Pilbratt G. L. et al., 2010, A\&A, 518, L1

Poglitsch A. et al., 2010, A\&A, 518, L2

Poulton C. J., Greaves J. S., Collier Cameron A., 2006, MNRAS, 372, 53

Quillen A. C., Thorndike S., 2002, ApJ, 578, L149

Reidemeister M., Krivov A. V., Stark C. C., Augereau J.-C., Löhne T., Müller S., 2011, A\&A, 527, A57

Rieke G. H. et al., 2004, ApJS, 154, 25 
Schütz O., Nielbock M., Wolf S., Henning T., Els S., 2004, A\&A, 414, L9

Scott K. S. et al., 2008, VizieR Online Data Catalog, 738

Scott K. S. et al., 2012, MNRAS, 423, 575

Shimizu I., Yoshida N., Okamoto T., 2012, MNRAS, 427, 2866

Smail I., Ivison R. J., Blain A. W., 1997, ApJ, 490, L5

Thureau N. D. et al., 2014, MNRAS, 445, 2558

Williams J. P., Andrews S. M., 2006, ApJ, 653, 1480

Wilson G. W. et al., 2008, MNRAS, 386, 807
Wyatt M. C., 2008, ARA\&A, 46, 339

Wyatt M. C., Dermott S. F., Telesco C. M., Fisher R. S., Grogan K., Holmes E. K., Piña R. K., 1999, ApJ, 527, 918

Wyatt M. C. et al., 2012, MNRAS, 424, 1206

Zechmeister M. et al., 2013, A\&A, 552, A78

This paper has been typeset from a $\mathrm{T}_{\mathrm{E}} \mathrm{X} / \mathrm{L} \mathrm{T}_{\mathrm{E}} \mathrm{X}$ file prepared by the author. 